by $\mathrm{Br}$ andon $\mathrm{Br}$ own, Jerome T. Galea, Kar ine Dube,

Peter Davidson, Kaveh Khoshnood, Lisa Holtzman,

Logan Marg, and Jeff Taylor

\title{
The Need to Track Payment Incentives to Participate in HIV Research
}

roviding incentives to research participants - in cluding money, gifts, and services - is an accepted and common practice in biomedical HIV re search.1 Here, we focus on financial incentives: "[p]ayment to research subjects for participation in studies."2 Although some studies suggest that altruism motivates many people to participate in research, 3 payment to participate is often essential for ensuring enrollment and retention in clinical research,4 including high-risk HIV biomedical trials, which may result in negative health outcomes.5While the payment offered across similar studies can greatly vary,6 surprisingly little research exists on the factors researchers consider when deciding on an appropriate payment amount, includ ing how to determine when an amount is "excessive" or constitutes an "undue inducement" to participate in a study. Further, the influence of incentives on partici pant recruitment, retention, and informed consent is virtually unknown, particularly in the biomedical HIV research field. We present one potential approach to explore the impact of financial incentives on biomedi cal HIV research and further clarify undue induce ment-namely, the development of a framework to support ethical decision-making regarding payment to participate, which is based on input from key stake holders and includes a database that allows for tracking payment practices.

\section{Concerns about Payment to Participate}

deally, incentives encourage participation and reten tion in clinical and behavioral research. However, payment to participate in research might negatively affect research findings or constitute an undue induce ment to enroll in risky research.7For example, in a large study conducted in the United States, $75 \%$ of

Brown B, Galea JT, Dube K, et al. The need to track payment incentives to participate in HIV research. IRB: Ethics \& Human Research 20i8;40(4):8- professional research participants (persons who can easily earn $\$ 40,000$ per year in studies) concealed information to qualify.8The same study found that some participants provided false information to stay in a study, hid contraindications, and, in some cases, in flicted self-harm to qualify.9 When involved in multiple simultaneous studies, such participants may harm the science of lower-paying studies by taking shortcuts to move on to studies with larger payment incentives.

Another concern related to the negative impact of financial incentives arises when participants perceive a link between study payment rates and the relative risk of study participation, with higher-paying studies considered higher risk than lower-paying studies. In a 2010 U.S.-based study, 10 researchers took participants through a series of hypothetical experiments. Each scenario gave participants the background information about the procedure and the amount they would be provided for participating (\$USD 25 or \$USD 1000). Participants were then asked to rate how risky they believed the study to be. Researchers found that as pay ment level increased, participants perceived the risk of the study to be much greater. The study authors argued that potential research participants might incorrectly assume studies with lower payment rates also present fewer health risks compared to higher-paying studies.11 Also concerning is that payment may distort sampling. The amount of payment or providing any payment at all may result in oversampling populations from low socioeconomic backgrounds who have different attributes compared to the broader population, poten tially distorting study outcomes. Thus, the true impact of incentives on recruitment, retention, and informed consent are unknown.

The U.S regulations governing research with hu mans require researchers to obtain informed consent "under circumstances ... that minimize the possibil- 
ity of coercion or undue influence," but the document does not define "undue influence" for practical use. 12 The ethics guidelines of the Council for International Organizations of Medical Sciences state that payment to participate must not cause undue inducement (see guideline 13), while they acknowledge that studies about financial incentives are needed to define "undue inducement." 13 And the Belmont Report, issued by the National Commission for the Protection of Human Subjects of Biomedical and Behavioral Research, says that "undue influence" in research must be avoided. The report describes undue influence as occurring "through an offer of an excessive, unwarranted, inap propriate or improper award or other overture in order to obtain compliance. Also, inducements that would ordinarily be acceptable may become undue influences if the subject is especially vulnerable." 14

Ezekiel Emanuel and colleagues contend that undue inducement is an offer of goods with gratuitous value that compels participants to use poor judgment while risking serious harm. 15 Yet even with this definition of undue inducement, investigators and institutional review boards (IRBs) find it difficult to know when a financial or other incentive to participate in research constitutes an undue inducement. For instance, a recent study in the U.S. asked 115 IRB members to identify undue inducement, which was defined as an offer of payment that distorts participants' ability to perceive accurately the potential risks and benefits of research, and $26 \%$ were unable to do so. 16 This illustrates that there is much work to be done to clarify when undue inducement occurs and to prevent undue inducements from being used to incentivize individuals to participate in research.

Even within the same study, differing economic circumstances may mean that a fixed payment amount constitutes undue inducement for one participant and reasonable compensation for another, so it may be pru dent to err on the side of what is best for people of low er socioeconomic status to avoid undue inducement. 17 In addition, the significance and potential effects of incentives may vary based on the vulnerability of par ticipants, and for HIV research, these may vary among subpopulations who are often stigmatized (e.g., gay and bisexual men, transgender people, people who inject drugs illegally, homeless youth). Participant judgment and potential harm are measurable variables, but with out a clear, consistent definition of what is excessive payment, we cannot differentiate between appropriate and inappropriate incentives, including those that exert an undue inducement. 18 If IRB members have difficulty identifying undue inducement, and if we have no data on incentives, making that distinction is a difficult task.

Some argue that IRBs are too focused on preventing undue inducement and that this focus has led to chroni cally low payments (i.e., "payment conservatism"), which means that participants may be unfairly compen sated for their socially valuable contribution and for accepting potential research risks and other researchrelated burdens. 19Thus, rather than striving only for incentives that avoid undue influence, researchers and IRBs must focus on promoting payments that are fair. A focus on providing fair incentives minimizes the risk of exploitation and increases the chance of recruiting a

\section{A}

database of payment practices in HIV research would provide baseline data that could be used for developing guidance on the range, types, and amounts of incentives that investigators should provide to participants in HIV studies.

diverse range of research participants.20 However, most researchers do not have a reference for comparison, which burdens researchers with trying to determine the appropriate and fair incentive for each project on a case-by-case basis. Rules for acceptable gifts, services, and payment should be written with consideration for study location and population, but this should not be done without a clearer understanding of currently offered incentives and how these practices align with ethical beliefs of appropriateness and fairness among key stakeholders. Otherwise, researchers may continue to follow perceived trends or historical precedents to determine what they perceive to be ethically appropri ate incentives without critical assessment.

\section{A Payment Database for HIV Research}

fforts to control over- and underincentivizing individuals to participate in research, as well as to address the issues of fairness, are under way in some countries. For example, South Africa developed standardized payments for participants (i.e., ZAR 150, or approximately \$USD 14),21 and Brazil prohibits all monetary payment for participation in clinical trials. 22 In the United States, the National Institutes of Health $(\mathrm{NIH})$ recently implemented an intramural grant policy 
that delineates baseline payment rates based on "in convenience units" (discomfort levels or inconvenience of the procedure) for research participants at the NIH Clinical Center. 25

Despite these initiatives, there is little empirical data about financial incentives used in clinical studies. Also lacking is written guidance on the range, types, and amount of payments to provide to research partici pants. Moreover, while investigators are required by IRBs to report payment to participants in their research protocols and informed consent documents, these incentives are not systematically tracked to permit comparison and reference, and incentive amounts may be reported inconsistently (e.g., by study visit, visit type, or for the entire study). Additionally, it is unclear whether IRBs routinely record incentives in separate data fields in their electronic database of investiga tors' applications for review of their protocols. And scholars interested in knowing about payment incen tives may look to ClinicalTrials.gov for such data only to be disappointed since investigators are not required to provide information about payment to participate when they register their study at this site.24 Academic journals also do not routinely ask for incentive infor mation, and most authors do not report this informa tion in publications about their studies.

In HIV clinical trials, participants may face greater than minimal risk (e.g., analytical treatment interrup tion), the prospect for direct individual benefit is low, and participants may face additional social vulnerabili ties (such as belonging to a sexual minority or having lower socioeconomic status), all of which may affect motivations to participate. 25 In addition, when basic health care is absent or difficult to obtain but included with participation in a high-risk clinical study, it may be viewed as an incentive to participate. A database of payment practices in HIV research would provide base line data that could be used for developing guidance on the range, types, and amounts of incentives inves tigators should provide to participants in HIV studies. Such a database could also help identify what types of payments in various contexts might constitute undue inducement to participate in HIV research.

The process for developing a payment database should include 1) determining how key stakeholders view and assess financial incentives, 2) reaching consen sus among these stakeholders on factors to consider when deciding on payment practices, 3) reviewing the literature on incentives provided in previous biomedical
HIV studies, and 4) developing a framework to foster ethical decision-making about incentives by investiga tors and IRBs. In particular, prospective, actual, and former participants in HIV research should be con sulted about their views and experiences with payment practices in research. For example, a recent study in Zimbabwe with 1008 participants revealed that they were dissatisfied with how decisions on incentives were reached without considering their expectations.26 When community advisory boards are involved in research studies, they could be consulted during the stage of developing research protocols to share their views about payment incentives. The availability of a payment database would provide an opportunity to see what payment incentives were used for similar studies conducted in similar and different contexts.

With a payments database in place, researchers could be required to include a justification statement for incentives in their IRB application, to record incen tive data in a separate field in the IRB applications, and to report payment information when they register their study on ClinicalTrials.gov or other venues that report study protocols and results. Studies proposing what are considered inadequate (possibly exploitative), excessive, or possibly undue incentives may be reviewed more carefully and not permitted to proceed in order to respect and protect study participants.

Developing a system that allows for tracking pay ment data in HIV studies will open new avenues for exploring topics such as incentive disparities, undue inducement, and failure or success rates of studies that require participants to remain incentivized over time. A payment incentive database could provide better guid ance and standardization for determining the appropri ate incentives based on past projects. Such a repository would generate a more ethically justified and scien tifically productive system for providing incentives, including optimal rates for recruitment and retention, and ranges of incentives used in similar studies to start the discussion on what payment amount is appropri ate. Furthermore, collection of payment incentive data could create transparency of incentivizing practices to help IRBs be more effective and consistent in reviewing protocols that include payment incentives. The tools developed for tracking payment incentives in HIV stud ies should be transferable to other areas of research, including cancer clinical trials, as well as early-phase and high-risk research with low prospect of direct clini cal benefits. 27 
It is possible that even with a payment incentive database, it may be difficult to reach consensus on definitions of inadequate, appropriate, or excessive in centives, not to mention harmonizing incentives within and across different types of studies. Nonetheless, a payment incentive database could allow each IRB or institution to explicate and apply its own policy consis tently across similar protocols with reference to a com monly recognized set of ethically salient parameters. 18 Researchers could also use the database generated from this research for secondary data analysis regarding cost-effectiveness of incentives in terms of maximizing recruitment and retention, topics that have not received much attention in the scientific literature.

\section{Conclusion}

ultiple factors influence decisions on which payment incentives to provide in research, but empirical research addressing the factors that should be considered when making these decisions is lacking. Without empirical research and reference data, we can not begin to examine this issue, much less define exces sive payments and undue inducements. Since payment to participate may have an effect on participant recruit ment and retention, and thus affect the move from a controlled study environment to real-world settings and applications, the lack of data about payment incentives represents a missing link in conducting ethical research. To address the gap in knowledge about payment practices, we have presented one potential method for further exploring the impact of incentives in biomedical HIV research and for defining and identifying undue inducement with regard to payment practices.

- Brandon Brown, PhD, MPH, is an assistant professor at the Center for Healthy Communities in the Division of Clinical Sciences in the School of Medicine at the University of California, Riverside; Jerome T. Galea, PhD, MSW, is a research associate in the Department of Global Health and Social Medicine at Harvard Medical School; Karine Dube, DrPH, is an assistant professor at the Gillings School of Global Public Health at the University of North Carolina, Chapel Hill; Peter Davidson, PhD, is an assistant professor in the Division of Global Public Health in the Department of Medicine at the University of California, San Diego; Kaveh Khoshnood, PhD, MPH, is an as sociate professor at the Yale School of Public Health; Lisa Holtzman, MPH, is a research assistant in the Department of Global Health and Population at the Harvard TH Chan School of Public Health; Logan Marg, MA, is a sociology PhD candidate in the Center for Healthy Communities in the School of Medicine at the University of California, Riverside; and Jeff Taylor is a community advisory board member for the Collaboratory of AIDS Researchers for Eradication, in Palm Springs, CA.

\section{Acknowledgment}

Brandon Brown and Lisa Holtzman were supported by
NIAID grant \#RoiAI 1146717 (HIV Cure Studies: Risk, Risk Perception, and Ethics).

\section{References}

1. Brown JA, et al. Effect of a post-paid incentive on response rates to a web-based survey. Survey Practice. 20i6;9(i):i-io; Lee R, et al. Incentivizing HFV/STI testing: A systematic review of the lit erature. AIDS and Behavior 20i4;i8(5):905-9i2; London A, Ripley B. Compensation or inducement? What IRBs need to know about paying subjects for participation. PRIM\&R Webinar 1017; Grady C. Payment of clinical research subjects. Journal of Clinical Investiga tion $2005 ; \mathrm{ii} 5(7)$ :i68i-i687.

2. U.S. Food and Drug Administration. Payment and reimburse ment to research subjects-information sheet. 2018. https://www.fda. gov/Regulatorylnformation/Guidances/ucmi 26429.htm.

3. Largent E. For love and money: The need to rethink benefits in HIV cure studies. Journal of Medical Ethics 2017;43:96-99; Arnold MP, Evans D, Vergel N. Recruitment and ethical considerations in HIV cure trials requiring treatment interruption. Journal of Virus Eradication 2.ox5; $\mathrm{x}(\mathrm{i}): 43 \sim 48$; Dube $\mathrm{K}$ et al. Willingness to participate and take risks in HIV cure research: Survey results from 400 people living with HIV in the US. Journal of Virus Eradication 20i7;3(i):40$50 ; \mathrm{e} 2 \mathrm{i}$.

4. See ref. 1, Grady 2005; Bentley JP, Thacker PG. The influence of risk and monetary payment on the research participation decision making process. Journal of Medical Ethics 2004:30(31:293-298.

5. Lo B, Grady C, on behalf of the Working Group on Ethics of the International AIDS Society. Ethical considerations in HIV cure research: Points to consider. Current Opinion in HIV and AIDS 20i3;8(3):243-249.

6. See ref. 1, Grady 2005; Ripley EB. A review of paying research participants: It's time to move beyond the ethical debate, journal of Empirical Research on Human Research Ethics 2००6; (4):9-20.

7. See ref. 4, Bentley and Thacker 2004; Booker CL, Harding S, Benzeval M. A systematic review of the effect of retention methods in population-based cohort studies. BMC Public Health 2011:11:249; Grant RW, Sugarman J. Ethics in human subjects research: Do incen tives matter? Journal of Medicine and Philosophy zoo 4; z9(6):yij738.

8. Devine EG et al. Concealment and fabrication by experienced research subjects. Clinical Trials 20 i3;io(6):935-948.

9. See ref. 8.

10. Cryder CE et al. Informative inducement: Study payment as a signal of risk. Social Science \& Medicine 20io;7o(3):45 5-464.

11. See ref. 10.

12. 45 CFR 46.116 .

13. Council for International Organizations of Medical Sciences, World Health Organization. International Ethical Guidelines for Bio medical Research Involving Human Subjects. Geneva, Switzerland: Council for International Organizations of Medical Sciences, 2002.

14. National Commission for the Protection of Human Subjects of Biomedical and Behavioral Research. The Belmont Report: Ethical Principles and Guidelines for the Protection of Human Subjects of Research. Washington, DC: Government Printing Office, 1979.

15. Emanuel EJ et al. Undue inducement in clinical research in developing countries: Is it a worry? Lancet 2005;366(9482):336-340. 16. See ref. 3, Largent 2017.

17. Davidson P, Page K. Research participation as work: Compar ing the perspectives of researchers and economically marginalized populations. American Journal of Public Health 20i2;i02(7):i2541259.

18. See ref. 7, Grant and Sugarman 2004.

19. Largent EA, Lynch HF. Paying research participants: The outsized influence of "undue influence." IRB: Ethics \& Human Research 2oi7;39(4):i-9-

20. See ref. 19.

21. Rhodes R, Gligorov N, Schwab AP. The Human Microbiome: Ethical, Legal and Social Concerns. Oxford: Oxford University Press, 


\section{3, PP- X*v>}

22. Lobato Let al. Impact of gender on the decision to partici pate in a clinical trial: A cross-sectional study. BMC Public Health 20 i 4 ; i 4 : i 56.

23. Department of Health and Human Services, Office of Human Subjects Research Protections. HRPP Standard Operating Procedurel Policy Approval \& Implementation: Recruitment, Selection and Compensation of Research Subjects. 2015. https://ohsr.od.nih.gov/ public/SOP_i3_v3_i 1-15-15_508.pdf.

24. Alemayehu D, Anziano RJ, Levenstein M. Perspectives on clinical trial data transparency and disclosure. Contemporary Clinical Trials 20i4;39(i):28-33.

25. See ref. 3, Largent 2017; see ref. 6, Ripley 2006; Presidential
Commission for the Study of Bioethical Issues. Moral Science: Pro tecting Participants in Human Subjects Research. Washington, DC: Presidential Commission for the Study of Bioethical Issues, 2011; Resnik DB. Bioethical issues in providing financial incentives to research participants. Medicolegal and Bioethics 2015;5:35-41.

26. Mduluza T et al. Study participants incentives, compensation and reimbursement in resource-constrained settings. BMC Medical Ethics 20i3;i4(suppl. 1):S4

27. Brown B et al. Transparency of participant incentives in HIV research. Lancet HIV 20i6;3(io):e456-e457.

28. Brown B, Merritt MW. A global public incentive database for human subjects research. IRB: Ethics \& Human Research $2^{\circ} \mathrm{i} 3 ; 35(2): \mathrm{i} 4-\mathrm{i} 7$. 